\title{
Design of a new high energy rear underrun protective device
}

\author{
F. Cappello, T. Ingrassia \& V. Nigrelli \\ University of Palermo, Dipartimento di Meccanica, Palermo, Italy
}

\begin{abstract}
In recent years the vehicle design field has followed a progressive evolution by focusing its main interest on passive and active safety levels. One of the most injurious cases for the safety of passengers is the crash between a car and a heavy vehicle (truck).

Generally, in fact, when such an occurrence happens, the greatest damage is suffered by the passengers of the car. This is because of the considerable structural difference between the two vehicles and the lack of devices to reduce the injuries of the car passengers. In this work, as a consequence of the previous considerations, we deal with the design of a new protective system for heavy vehicles over 3.5 tons. The new rear underrun protective device presented here, compared to the traditional one, can dissipate a greater amount of energy; this reduces the damage suffered by the passengers of a car involved in an impact with a truck. To simulate the behaviour of the new protective device during different kinds of crashes, dynamic finite element method (FEM) analyses have been carried out. The comparison between the new protective system and the standard one has shown that the new designed protective system has better performances in terms of energy absorption and peaks of decelerations.
\end{abstract}

Keywords: RUPD, FEM, crash analyses.

\section{Introduction}

Usually when a road accident between a car and a heavy vehicle happens, all the protection features for the occupants built into the car, such as seatbelts and airbags, have a reduced effectiveness. This because of the very big differences in geometry and stiffness between the two vehicles. The very large height of the truck, especially when the heavy vehicle is not equipped with a suitable 


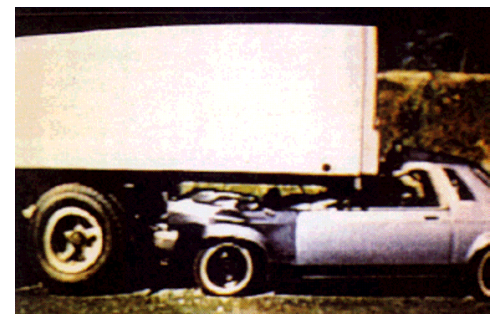

Figure 1: Underride of a car.

rear underrun protective device (RUPD), in fact, can allow the underride of the car (figure 1).

For this reason many studies and regulations have been developed during recent years. The Directive Council 70/221/EEC [1], based on ECE Regulation no. 58 [2], has defined all the requirements for the rear underrun protection systems for trucks and trailers with a total mass of more than 3.5 tons.

A study of $58 \mathrm{car} /$ truck collisions [3] has shown that, generally, the rear underrun protection systems are ineffective because of the large ground clearance.

First estimates of this study have in fact shown that by limiting the RUPDs height to $400 \mathrm{~mm}$ it may be possible to considerably reduce the damage suffered by the passengers of a car involved in a rear side impact.

In addition, it was found that the social costs for fatalities and severe injuries could be reduced from 78 million to 69 million of euros.

An effective rear underrun protective device therefore must be able to contain the intrusion and to dissipate high energy with tolerable decelerations in the passengers' compartment of a striking car.

Following the previous guidelines in the study presented here, a new RUPD has been designed, called HED-SafeBar (High Energy Dissipation Safe Bar), which able to increase the level of crashworthiness between a truck and a passengers' car; this goal has been reached by introducing some specific components, called crashboxes, which are able to absorb a great amount of energy thanks to their large deformations.

The comparison with a standard rear underrun protective system has shown very remarkable improvements in terms of absorbed energy, peaks of deceleration and underride of the car, with a very considerable increase in the safety level of the passengers.

\section{The rear underrun protective devices (RUPDs)}

As already mentioned, in most cases when a car crashes into a truck, the car suffers the most remarkable damage. This is essentially due to the existing notable differences of mass and geometry between the two vehicles. Particularly, the ground clearance of the industrial vehicle floor, being comparable to the height of a car, can cause the underride of the car. 
Likewise, in many instances, because of the excessive stiffness of some components of the standard RUPDs (the stirrups), all (or almost all) the kinetic energy before the impact is wasted from the car and this can have dramatic repercussions on the passengers, because of the very high values of deceleration.

So the main requirements of an optimal rear underrun protective system are essentially two: avoiding the underride of the car and absorbing the greatest amount of energy so as to reduce the deceleration peaks and, consequently, the injuries suffered by the passengers.

In any designing or redesigning process of a new device it is also necessary to respect the regulations governing its use. In Europe the ECE R 58 [2] regulates the design of RUPDs for all the industrial vehicles with mass greater than 3500 $\mathrm{kg}$. These regulations impose that (fig. 2):

- $\quad$ the RUPD width, compared to the rear axle length, must not be longer or shorter by more than $100 \mathrm{~mm}$ on every side;

- $\quad$ the height of the RUPD profile must not be less than $100 \mathrm{~mm}$;

- $\quad$ the RUPD must be put on the back end of the vehicle;

- $\quad$ the ground clearance must not be higher than $550 \mathrm{~mm}$.

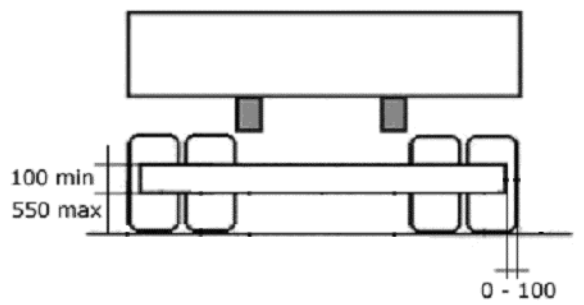

Figure 2: Dimensional limits of a RUPD.

\section{Some examples of rear underrun protective devices}

The more common (standard) rear underrun protective system is essentially constituted of two stirrups and a crossbar (fig. 3).

The stirrups are used to rigidly connect the RUPD to the longitudinal beams of the truck frame by means of bolts or welding.

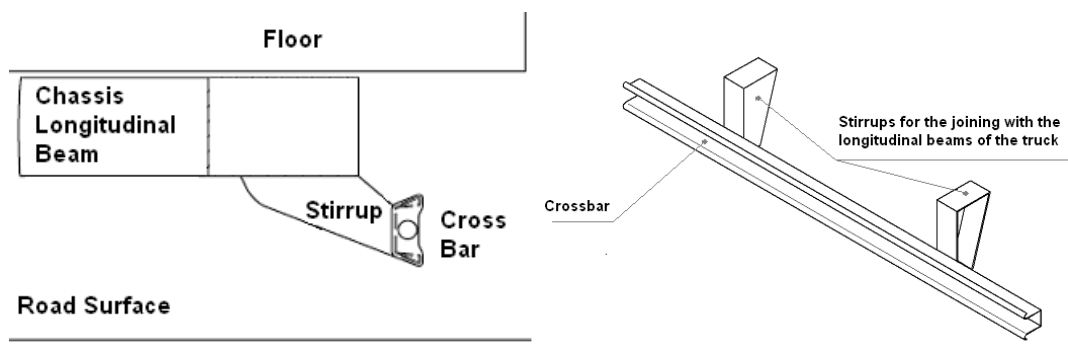

Figure 3: $\quad$ Scheme of the standard RUPD. 
Generally the crossbar section of this kind of RUPD has a shape like a trapezium (fig.3); the crossbar, moreover, is often where the rear lights of the truck are fixed.

Nevertheless, even if this kind of device is nowadays widely used, many different prototypes have been developed during recent years [4-6] in order to improve the performance of the standard RUPD and, consequently, reduce the decelerations suffered by the passengers and avoid the underride of the car in the case of a crash.

In previous papers $[7,8]$ a suitable system has been proposed to reduce the intrusion into the passengers' compartment of a striking car, by using a set of energy absorbers.

Two of the most innovative protective systems are shown in figures 4 and 5.

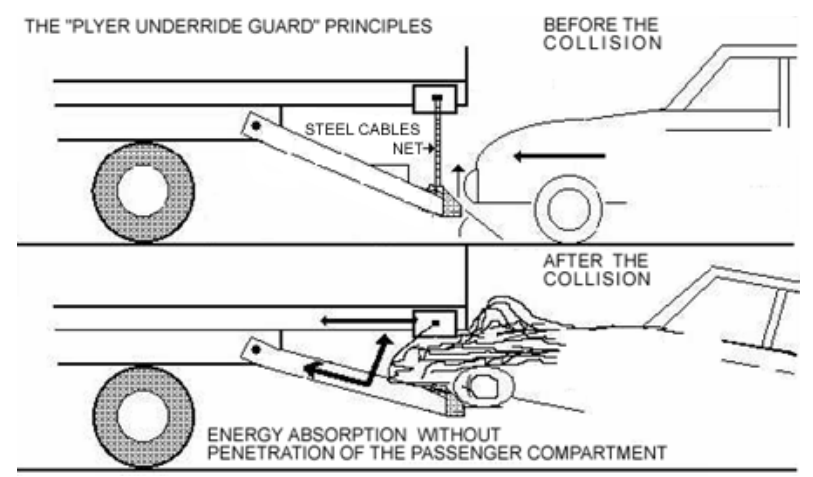

Figure 4: Working scheme of the Plier Underride Guard.
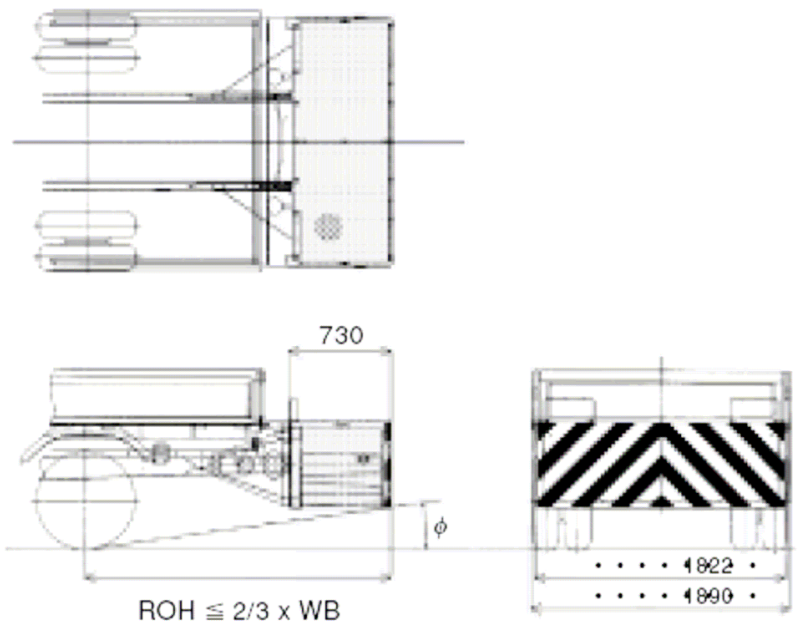

Figure 5: The RUPD developed from Nagaike. 
The first one (fig. 4), the so-called "Plier Underride Guard", developed from the Unicamp State University (Brazil) [4], is based on the mechanical principle of a simple pliers tool. In case of collision, the car's front bumper impacts against a net of steel cables, stretching the cables and, consequently, tending to lift the lower bar (fig. 4). The front of the car is so "bitten" by the lower bar and the chassis of the truck, as if by pliers; the compression of the car front end avoids the wedge effect and, consequently, the car underride.

The second one has been developed by Nagaike of the Truck and Bus Development Department of the Japanese Transport Department [9].

The device is composed of eight tubular energy absorbers linked to the rear side of the truck and a rectangular plate (fig. 5).

During the impact most of the kinetic energy is absorbed by means of tubular elements deformation.

However a limit to the use of this system is represented by the excessive longitudinal overall dimensions that make it unsuitable to equip a standard heavy vehicle.

\section{Design of the HED-SafeBar}

The new rear underrun protective device introduced in this paper is composed of different components; among these the principal ones are (fig. 6):
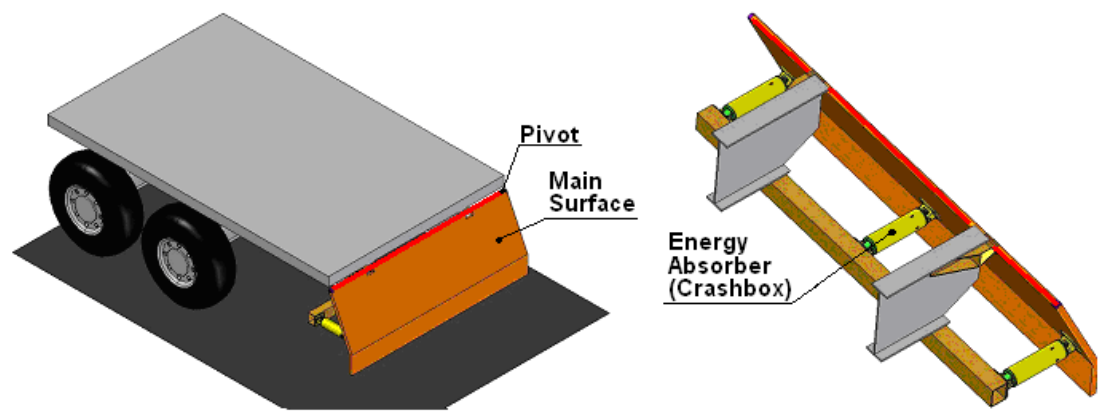

Figure 6: $\quad$ Scheme of the HED-SafeBar.

- the main surface; it represents the contact surface and is used to make the crashboxes collapse and prevent the underride of the car. The main surface has been reinforced with an internal net of steel beams, so as to allow a good crushing of the crashboxes;

- a set of three crashboxes; they represent the energy absorbers of the system. Every crashbox is composed of a tube of aluminium 6061 T6 filled with closed cell metallic foam (Cymat A35620SC 030SS).

Moreover, to reduce the buckling load peak and so make the beginning of the structure collapse easier, four collapse starters (buttonholes) have been introduced on the surface of the tube.

The crashboxes are connected to the main surface and the hollow bar (connected to the truck chassis) by means of two spherical joints. Thanks to this 
joining solution every crashbox is stressed exclusively along its longitudinal axis, avoiding, in this way, any instability effect on the structure. Moreover, to be sure the crashbox is not submitted to any bending effects, besides the spherical joints, a telescopic guide has been inserted inside the tube (fig. 7).

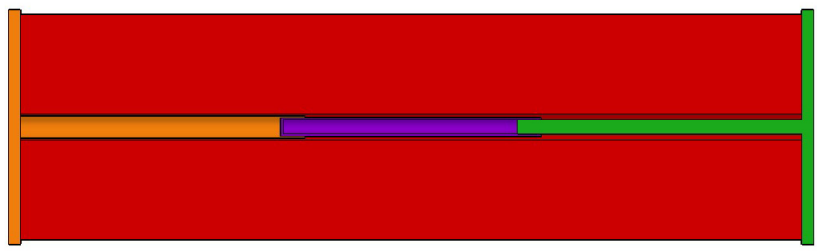

Figure 7: Outline of the internal part of a crashbox.

The tubes of the crashboxes have a circular section. This kind of section has been selected because it allows, as demonstrated in [10], to waste a greater amount of energy in comparison with other kinds of section.

The working principle of the HED-SafeBar is the following: when a car rams into the rear side of a truck, it pushes the main surface that rotates around the pivot (fig. 6) placed on the highest extremity of the main surface; as a consequence, the energy absorbers (crashboxes) are crushed and can disperse a great amount of energy through their plastic deformations. Moreover, the main surface prevents the car underriding.

\section{Crash numerical simulations}

To simulate the working performance of the new HED-SafeBar and to compare it with the standard RUPD, an explicit finite element method (FEM) code has been used. In this way the real conditions during the collision between a car and a heavy vehicle have been simulated through dynamic numerical analyses by using the Ansys-LsDyna software. Because of the characteristics of the numerical crash simulation, in which there are large deformations of the components in plastic range, it has been necessary to carry out nonlinear analyses.

To set up this kind of analyses the FEM models of a truck and a car have been carried out. The FEM models of the truck, the HED-SafeBar and the standard RUP have been carried out at the University of Palermo. The FEM model of the car has been provided by the NCAC (National Crash Analysis Center) [11] of the George Washington University.

The car is a Geo Metro 1998 and is composed of more than 200 different parts modelled with shell and solid elements and 20 different material types (fig. 8).

As regards the truck FEM model, to reduce the analyses running times, only the rear side (that involved in the crash) has been used and it has been equipped both with the HED-SafeBar (fig. 9(a)) and the standard RUPD models (fig. 9(b)). Moreover, for the same reason, a simplified model of the truck, only composed of the chassis, the floor, the axles and the tyres, has been carried out. 


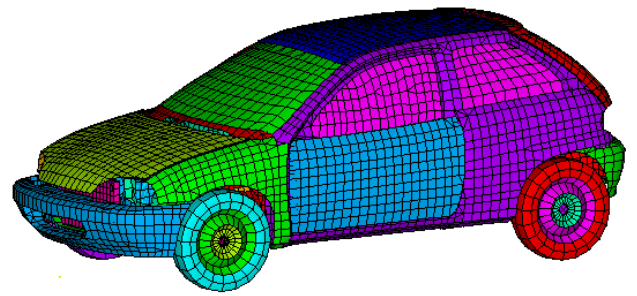

Figure 8: $\quad$ FEM model of the car.
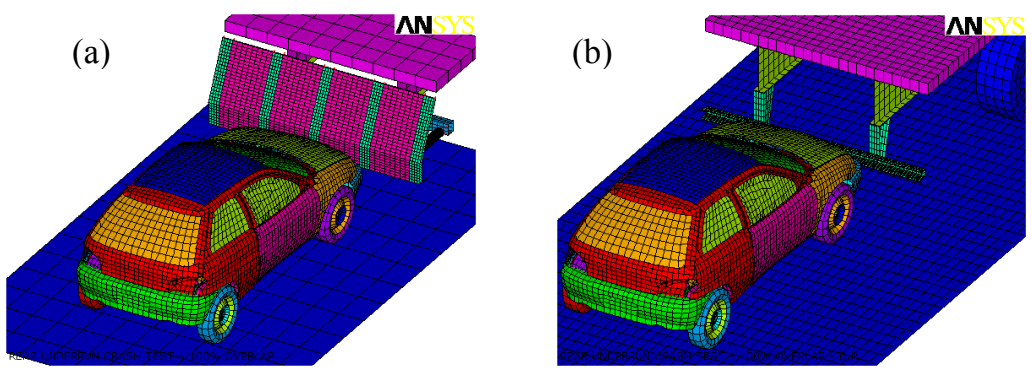

Figure 9: The FEM model of the rear side of a truck equipped with the HED-SafeBar (a) and the standard RUPD (b).
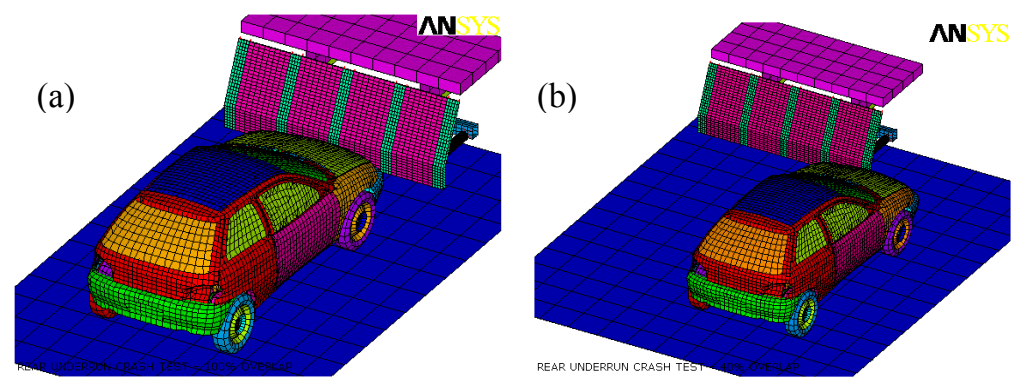

Figure 10: The $100 \%$ overlap (a) and the $40 \%$ overlap (b) crashes.

To avoid the hourglass phenomena and, therefore, to be sure of the reliability of the test results, a full-integral formulation has been used for all the elements of the front side of the car.

To measure and plot the decelerations during the crash, six accelerometer elements have been placed on the car in correspondence of the top of engine, the left rear sill, the right rear sill, the floorpan, and the anterior left and right brakes.

To evaluate the behaviour of the new system in different working conditions, two kinds of test have been simulated (fig. 10); in both cases the car impacts against the rear side of the heavy vehicle at $56 \mathrm{~km} / \mathrm{h}$ but in the first kind of crash (called 100\% overlap) all the front surface of the car is involved in the impact, whereas in the second one (called $40 \%$ overlap) only $40 \%$ of the car front surface crashes into the rear part of the truck. 


\subsection{Optimization of the crashboxes}

Before studying the working performance of the HED-SafeBar, a set of FEM analyses, to identify the optimal shape of the crashboxes, has been carried out.

Starting from the draft scheme of the crashbox shown in figure 7 , some preliminary simulations have been performed to understand the influence of all the geometric parameters on the values of absorbed energy and peak of deceleration. As consequence of these preliminary analyses, it has been chosen to run an optimization process by varying the followings parameters (fig. 11):

-the orientation of the buttonholes (longitudinal or transversal);

-their distance $(Z)$ from the extremity of the tube;

-their dimensions (H and $\mathrm{L}$ );

-the thickness of the pipe (S).

The obtained results are summarized in table 1 .
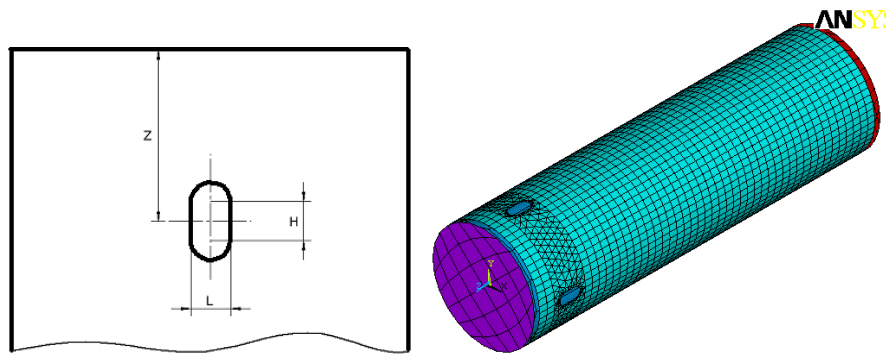

Figure 11: On the left, some of the optimization parameters of the crashbox; on the right the FEM model of the crashbox.

Table 1: Optimal values of the crashbox geometric parameters.

\begin{tabular}{|l|l|l|l|l|}
\hline Variable & S & Z & H & L \\
\hline Value & $1 \mathrm{~mm}$ & $60 \mathrm{~mm}$ & $10 \mathrm{~mm}$ & $10 \mathrm{~mm}$ \\
\hline
\end{tabular}

With regard to the orientation of the buttonholes, the longitudinal direction has turned out to be the best solution, not only in terms of overall absorbed energy during the crash but, above all, for the smaller buckling loads and, consequently, the smaller deceleration peaks.

\section{Results}

All the dynamic FEM simulations have shown the new proposed device, both in the case of $100 \%$ overlap crash (fig. 12) and in the $40 \%$ overlap impact, has better performance in comparison with the standard RUPD.

The obtained results demonstrate that the new system does not allow, in any case, the underride of the car; moreover, the measured decelerations are globally lower than the values obtained with the standard device, so reducing the possibility of serious injuries suffered by the passengers of the car. 


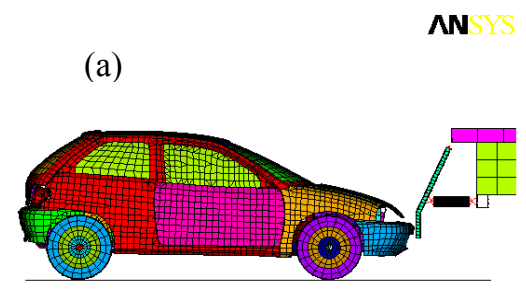

(b)

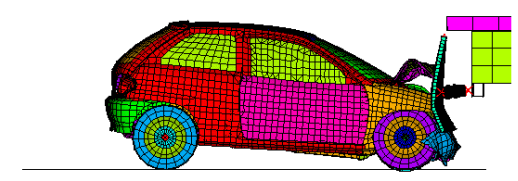

REAR UNDERRUN CRASH TEST - $100 \%$ ONERLAP - RUNO1

REAR UNDERRUN CRASH TEST - $100 \%$ ONERLAP - RUNO1

Figure 12: The HED-SafeBar before (a) and after (b) the frontal crash.

Table 2: $\quad$ Results of the $100 \%$ overlap FEM simulations.

\begin{tabular}{|c|c|c|c|c|c|c|}
\hline \multirow{2}{*}{} & \multicolumn{5}{|c|}{ Reduction in decelerations using the new RUPD in the 100\% overlap crash test } \\
\cline { 2 - 7 } & Left R.S. & Right R.S. & Floorpan & T. Engine & L. Brake & R. Brake \\
\hline Absolute (g) & -14.98 & -16.33 & -12.54 & -8.12 & -26.96 & -37.45 \\
\hline Perceptual & $33.75 \%$ & $32.91 \%$ & $27.18 \%$ & $11.48 \%$ & $37.81 \%$ & $51.45 \%$ \\
\hline
\end{tabular}

Table 3: $\quad$ Results of the $40 \%$ overlap FEM simulations.

\begin{tabular}{|c|c|c|c|c|c|c|}
\hline \multirow{2}{*}{} & \multicolumn{5}{|c|}{ Reduction in decelerations using the new RUPD in the 40\% overlap crash test } \\
\cline { 2 - 7 } & Left R.S. & Right R.S. & Floorpan & T. Engine & L. Brake & R. Brake \\
\hline Absolute $(\mathrm{g})$ & +8.36 & +2.44 & -2.38 & +24.08 & -44.47 & +4.30 \\
\hline Perceptual & $+35.58 \%$ & $+10.96 \%$ & $-8.13 \%$ & $+96.38 \%$ & $-54.03 \%$ & $+22.57 \%$ \\
\hline
\end{tabular}

The main results are summarized in tables 2 (100\% overlap) and $3(40 \%$ overlap).

It is possible to note that because of the greater stiffness of the side part of the new system (caused by the internal stiffening net of the main surface) compared to the traditional one, in the $40 \%$ overlap impact and for just some accelerometers, there is an increasing of the measured decelerations (table 3 ).

Nevertheless, this result does not invalidate the very good overall improvements obtained with the HED-SafeBar. Even if there is an increase (especially in the top of the engine), the maximum peak of the measured decelerations by using the new proposed RUPD is lower than the standard one. In the $40 \%$ overlap test, in fact, the maximum measured values are $-48 \mathrm{~g}$ on the top of the engine for the new system and $-82 \mathrm{~g}$ on the left brake for the standard device.

In both types of impact, the new device is able to absorb a greater amount of energy in comparison with the standard system, thanks to the big deformations of the crashboxes; this limits the crushing of the frontal part of the car and so protects the survival passengers' cell (fig. 13 - table 4).

\section{Conclusions and future work}

The HED-SafeBar has meets all the requirements for the design of a new rear underrun protective system. 


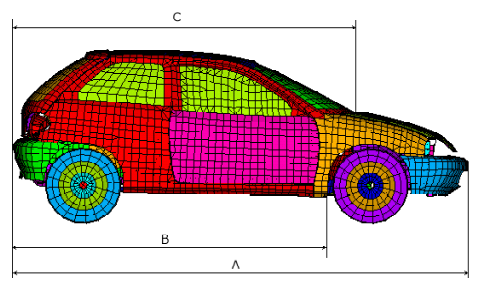

Figure 13: Values used to measure the crushing of the survival cell.

Table 4: $\quad$ Measured values of the survival cell.

\begin{tabular}{|c|c|c|c|}
\hline \multirow{2}{*}{$\begin{array}{c}\text { Variation of the } \\
\text { car dimensions }\end{array}$} & \multicolumn{3}{|c|}{ Crushing of the car [mm] } \\
\cline { 2 - 4 } & HDP-SafeBar & Standard RUPD & Variation \\
\hline$\Delta \mathrm{A}$ & 496.53 & 519.06 & -22.53 \\
\hline$\Delta \mathrm{B}$ & 29.87 & 40.85 & -10.98 \\
\hline$\Delta \mathrm{C}$ & 12.57 & 33.37 & -20.80 \\
\hline
\end{tabular}

In particular the new device:

- avoids the insertion of the car under the frame of the heavy industrial vehicle; - limits, as far as possible, the decelerations weighing on the passengers of the car;

- reduces the deformation of the survival cell of the car, thanks to the very large deformations of the energy absorbers of the protective device.

The new RUPD, in fact, is able to absorb more energy than the standard one: about $+89.4 \%$ in the $100 \%$ overlap crash $+20 \%$ in the $40 \%$ overlap impact.

A possible restriction to the use of the new device is its weight, which is slightly greater than the standard device (by about $38 \%$ ).

The proposed device offers, however, a strong alternative to the traditional systems, in comparison to which it has, of course, a greater level of quality and safety that could limit the number, unfortunately nowadays still very elevated, of victims in the road accidents.

One aspect of future work will be related to the study of a new solution that makes use of crashboxes that employ the outflow of a fluid; this system could result in more regular energy absorption.

\section{References}

[1] "Priorities for EU motor vehicle safety design", European Transport Safety Council, Brussels 2001

[2] ECE Regulation No. 58: "Uniform provisions concerning the approval of rear underrun protective devices (RUPD)"

[3] European Enhanced Vehicle-Safety Committee (EEVC), "Accident Analysis of Rear Underrun Accidents - Statistical Report and Preliminary Benefit Study. Working Group 14 Report"

[4] Mariolani J.R.L., Arruda A.C.F., Schmutzler L.O.F. "Development of new underride guards for enhancement of compatibility between trucks and 
cars", Proceedings of the 17th International Technical Conference on the Enhanced Safety of Vehicles, Paper No. 425, Amsterdam, The Netherlands, 2001

[5] Hope F., "Safety rear underride guards with an optional reverse impact braking system", Hope Technical Developments Ltd, United Kingdom, paper number 98-S4-P-2; http://www-nrd.nhtsa.dot.gov/

[6] Beermann H.J., "Behaviour of passenger cars on impact with underride guards". Int. J. of Vehicle Design, vol. 5, pp. 86-103, 1984

[7] Buttita R., Cappello F., Nigrelli V., "Dispositivo paraincastro posteriore ad elevato assorbimento energetico", XXIV AIAS conference, Milan 2005

[8] Buttita R., Cappello F., Nigrelli V., "Aumentare la sicurezza passiva", Progettare n.305, November 2006, 35-38

[9] Nagaike N., Hayashida T., Mizuno S., "Energy absorbing front frame structure for a vehicle", http://www.patentstorm.us/patents/6938948fulltext.html

[10] Buscemi G., Cappello F., Nigrelli V., "Crash behaviour of thin-walled columns varying the cross section shape and the velocity", www.ncac.gwu.edu 